\title{
US signs contract with ZMapp maker to accelerate development of the Ebola drug
}

\author{
Michael McCarthy
}

Seattle

The US government has signed a multimillion dollar contract with the maker of the experimental Ebola drug ZMapp to accelerate the drug's development.

Under the contract the US Biomedical Advanced Research and Development Authority (BARDA) will provide funding, expertise, and technical support to the drug company, Mapp Biopharmaceutical, of San Diego, California. BARDA is the US agency tasked with developing medical

countermeasures-including vaccines, medicines, diagnostics, and medical equipment - to respond to chemical, biological, radiological, or nuclear incidents or attacks, as well as pandemics and emerging infectious diseases.

The agreement was announced on 2 September by the US Department of Health and Human Services' Office of the Assistant Secretary for Preparedness and Response, which oversees BARDA. The initial contract will provide Mapp with $\$ 24.9 \mathrm{~m}$ (£15.1m; €18.9m) over 18 months to support work toward acquiring US Food and Drug Administration approval of the drug. The contract can be extended to a maximum of $\$ 42.3 \mathrm{~m}$.

Ebola is a filovirus that causes severe hemorrhagic fever with a high mortality rate. In some outbreaks the case fatality rate has reached $90 \%$. Since it was first detected in March, the current Ebola outbreak in west Africa has claimed more than 1550 lives, and there are no approved therapies or vaccines.

ZMapp is a combination of three humanized monoclonal antibodies, produced in genetically modified tobacco plants, which target three Ebola virus glycoprotein epitopes. The drug has been shown to provide a survival benefit in nonhuman primates experimentally infected with the virus. ${ }^{12}$ Last week, researchers reported that ZMapp was able to rescue rhesus macaques that had developed advanced disease as indicated by elevated liver enzymes, mucosal hemorrhages, and generalized petechiae. $^{3}$

Although it has never been tested in humans, the drug has been administered on an emergency basis to a number of patients with Ebola in recent weeks. Five of these patients have survived, and two have died.

As part of the new contract Mapp Biopharmaceutical will manufacture a small amount of the drug for early stage clinical safety studies that are needed to demonstrate the drug's safety and efficacy. Mapp also will work with BARDA to refine its manufacturing process to increase production yields.

BARDA is seeking additional proposals for the advanced development of antibody treatments, antiviral drugs, and vaccines against Ebola and the Marburg virus, another cause of viral hemorrhagic fever.

thebmj.com News: WHO gives go ahead for experimental treatments to be used in Ebola outbreak (BMJ 2014;349:g5161, doi:10.1136/bmj. g5161)

1 Olinger GG Jr, Pettitt J, Kim D, et al. Delayed treatment of Ebola virus infection with plant derived monoclonal antibodies provides protection in rhesus macaques. Proc Natl Acad Sci USA 2012;109:18030-5.

2 Pettitt J, Zeitlin L, Kim H, et al. Therapeutic intervention of Ebola virus infection in rhesus macaques with the MB-003 monoclonal antibody cocktail. Sci Trans/ Med 2013;5:199ra113.

3 Qiu X, Wong G, Audet J, Bello A, Fernando L, et al. Reversion of advanced Ebola virus disease in nonhuman primates with ZMapp. Nature 2014; published online 29 August. doi:10.1038/nature13777.

Cite this as: BMJ 2014;349:95488

๑ BMJ Publishing Group Ltd 2014 